\title{
Comparative bioinformatics analysis of prion proteins isolated from reptile, rodent, ruminant, and human species
}

\author{
Insung Ahn ${ }^{1}$ and Hyeon Seok Son ${ }^{2,3,4}$ \\ ${ }^{1}$ Bioinformatics Team, Supercomputing Center \\ Korea Institute of Science and Technology Information \\ Daejeon 305-806, Korea \\ ${ }^{2}$ Laboratory of Computational Biology and Bioinformatics \\ Institute of Health and Environment \\ Graduate School of Public Health \\ Seoul National University \\ Seoul 110-799, Korea \\ ${ }^{3}$ Interdisciplinary Graduate Program in Bioinformatics \\ Seoul National University \\ Seoul 151-742, Korea \\ ${ }^{4}$ Corresponding author: Tel, 82-2-740-8864; \\ Fax, 82-2-762-9105; E-mail, hss2003@snu.ac.kr
}

Accepted 1 November 2007

Abbreviations: PrP, prion protein; MD, molecular dynamics

\begin{abstract}
Prion proteins (PrPs) are infectious pathogens that cause a group of invariably fatal, neurodegenerative diseases, including Creutzfeldt-Jakob disease, by means of an entirely novel mechanism. They are produced by various species, including reptile, rodent, ruminant and mammals, during normal metabolic processes, but they can be slowly changed into pathogenic isoforms upon contact with other infectious PrP isoforms. This transmission can occur across species barriers. In the present study, phylogram for each PrP sequence was generated by PAUP* 4.0 program using Neighbor-Joining method with 1,000 times bootstrapping process for the phylogenetic analysis. The molecular dynamics (MD) simulations were performed by the SANDER module in the AMBER 7 package using Amber 99 force field. All the simulation process was conducted in the IBM p690 Supercomputing System in Korea Institute of Science and Technology Information. To reduce the calculation time, we used 'the Generalized Born (GB) model'. We compared the sequences and structural characteristics of normal and pathogenic (E200K) human PrPs with those of other reptile, rodent, ruminant and mammalian PrPs. Phylogenetic analysis revealed that, although the turtle PrP sequence is the most distinct of the PrPs analyzed, it
\end{abstract}

nonetheless retains five conserved secondary structural elements that are similar to those found in the mammalian PrPs, suggesting that these elements have important functions in vivo. The RMS deviation between the normal and E200K human PrPs was larger than that between the normal human and bovine PrPs, and all of the $\beta$-sheet structures in human E200K PrP were very stable during MD simulations.

Keywords: computational biology; neurodegenerative diseases; phylogeny; prions; proteomics

\section{Introduction}

Prion proteins (PrPs) are infectious pathogens that cause a group of invariably fatal, neurodegenerative diseases by means of an entirely novel mechanism. In mammals, the disease-causing isoform $\left(\mathrm{PrP}^{\mathrm{Sc}}\right)$ reproduces by recruiting and stimulating structural changes in the normal, cellular isoform $\left(\mathrm{PrP}^{\mathrm{C}}\right) . \mathrm{PrP}^{\mathrm{C}}$ is rich in $\alpha$-helical content and has little $\beta$-sheet structure, whereas $\operatorname{PrP}^{\mathrm{Sc}}$ has a smaller amount of $\alpha$-helical content and a large amount of $\beta$-sheet structure (Prusiner, 2004). Many scientists have tried to determine the exact function of cellular PrP in biological systems, but it remains unknown. Qin et al. (2002) suggested that it participates in cellular uptake or efflux of extracellular ligands, and might interact with copper ions. PrP binds copper with low-micromolar affinity and in a $\mathrm{pH}$-sensitive manner via $\mathrm{N}$-terminal histidine-containing octapeptide repeats (Jackson et al., 2001; Kramer et al., 2001; Burns et al., 2002). Binding of $\mathrm{Cu}^{++}$to the C-terminal portion of PrP in vivo seems unlikely, but it has been suggested by experiments using amino-terminal truncations of the protein (Burns et al., 2003).

The first study of prion diseases was conducted by Klatzo, Gajdusek, and Zigas in 1959, who discovered that the neurological symptoms of Creutzfeldt-Jakob disease (CJD) were very similar to those of Kuru, a disease found among the Fore native people in Papua New Guinea. Kuru was believed to be transmitted by ritualistic cannibalism (Gajdusek, 1977; Alpers, 1979). A few years later, Hadlow reported that Kuru resembles scrapie, a disease of sheep, and its long latent period led him to suggest that the responsible pathogen was a "slow virus" (Hadlow et al., 1982). In 1997, Prusi- 
ner reported that the pathogenic PrP that induces mad cow disease does not reproduce through transmittal of genetic information; instead, he proposed that $\mathrm{PrP}^{\mathrm{Sc}}$ reproduces by initiating changes in the structure of $\mathrm{PrP}^{\mathrm{C}}$, converting it to $\mathrm{PrP}^{\mathrm{Sc}}$. In the absence of competing evidence, his hypothesis has been generally accepted.

Fewer than $1 \%$ of human prion diseases appear to have an infectious etiology, whereas $\sim 85 \%$ occur spontaneously, and the remaining cases occur through dominant inheritance of germ-line mutations of the PrP gene (Prusiner, 2004). Among the inherited prion diseases with a CJD phenotype, the epidemiology of CJD ${ }^{\mathrm{E} 200 \mathrm{~K}}$ is of particular interest. According to Kahana (1974) and his colleagues, the largest cluster of CJD ${ }^{E 200 K}$ occurs among Jews of Libyan and Tunisian origin. This population experiences a 100-fold increased incidence of CJD, as compared to other populations. Several years later, the cause of this disease was determined to be genetic, resulting from an E200K mutation in PrP, and the disease was designated as familial CJD (Cathala et al., 1985; Nisipeanu et al., 1990; Zilber et al., 1991). The clinical symptoms of $\mathrm{CJD}^{\mathrm{E} 200 \mathrm{~K}}$ resemble the typical form of spontaneous CJD; patients present cognitive impairment and psychiatric changes $(80-83 \%$ of patients), cerebellar signs (43-55\%), visual signs $(19 \%)$, and myoclonic jerks (12\%) (Brown et al., 1991; Parchi et al., 1999). The mean age at onset in patients heterozygous for the E200K mutation is 58 years, and the mean duration is 6 months (Simon et al., 2000).

In performing routine histopathological examinations of cow brains from dairy farms in southern England, the UK Veterinary Laboratories Agency (formerly the Central Veterinary Laboratory) of Weybridge found critical evidence suggesting a new neurological disease of cattle, subsequently termed bovine spongiform encephalopathy (BSE). The neurohistological changes of BSE bear a high degree of resemblance to those of scrapie, the historical archetype of the transmissible spongiform encephalopathies (Wells et al., 1987). BSE has been transmitted experimentally by parenteral routes from cattle to other cattle (Dawson et al., 1990), to mice (Fraser et al., 1992), to pigs (Wells et al., 2003), and to cynomolgus macaques (Lasmézas et al., 1996). A considerable body of evidence also indicates that BSE has been transmitted, by natural or accidental means, via foodstuffs, to several other animal species and to humans (Kirkwood and Cunningham, 1994; Hill et al., 1997).

Because prion transmission occurs across species barriers, we conducted a comparative phy- logenetic analysis of $\mathrm{PrP}$ sequences from four different species (reptile, rodent, ruminant, and human). We also performed a comparative structural analysis of these PrPs based on molecular dynamics (MD) simulations.

\section{Materials and Methods}

\section{Protein sequences and phylogenetic analysis}

Phylogenetic trees for PrPs from 8 species including turtle (1U5L), mouse (1AG2), bovine (1DWY), human (1HJM), human E200K (1FKC), hamster (1B10), sheep (1XYU) and elk (1XYW) were created by the program PAUP* 4.0 (Swofford, 1999) using the Neighbor-Joining (NJ) method with bootstrapping of 1000 iterations. All the amino acid sequences were collected from Protein Data Bank database. Each branch length represents the phylogenetic distance. TreeView (Page, 1996) and Adobe Illustrator CS2 were used for graphical editing, and all the analyses were performed on Linux and Windows XP operating systems (Figure $1)$.

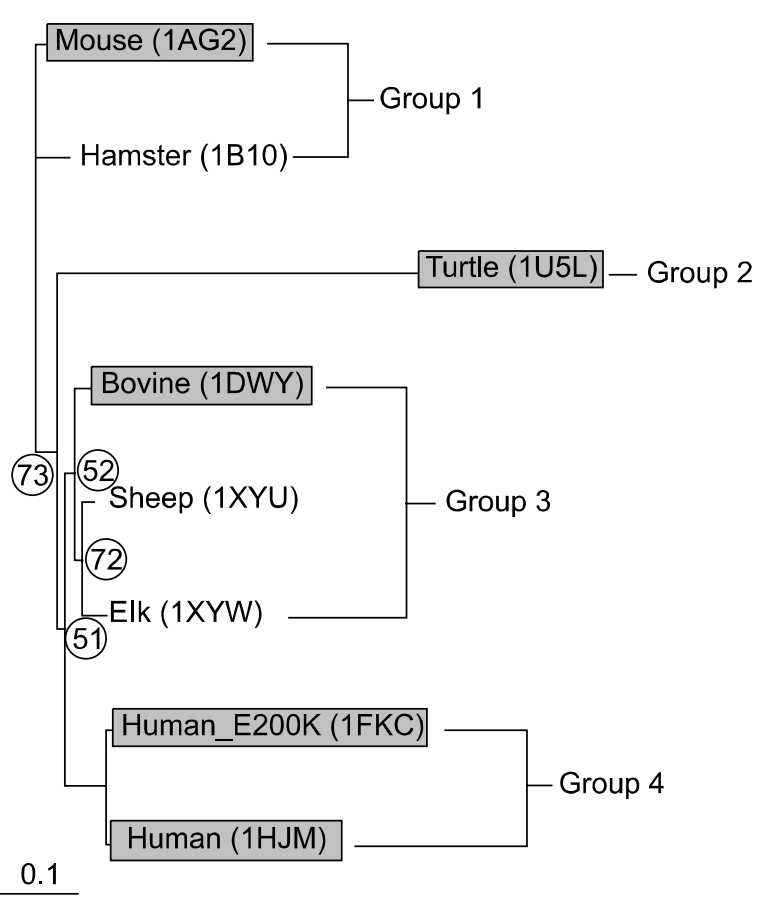

Figure 1. Phylogram of PrP sequences from turtle, mouse, hamster, bovine, sheep, elk and human (normal and E200K). Species names and PDB ID numbers are shown. Trees were produced by the PAUP* 4.0 program using the Neighbor-Joining method with bootstrap analysis of 1,000 iterations. Branch lengths are drawn to scale (bar $=0.1$ amino acid replacements per site). Bootstrap values (\%) not equal to $100 \%$ are represented as circled numbers in each node. 
Among 8 sequences which were mentioned above, 5 species from each node including turtle, mouse, bovine, human and human E200K were collected for further analysis. Protein sequences as well as NMR coordinate information for each PrPs was collected from the Protein Data Bank (PDB, http://www.rcsb.org/pdb/). The PDB ID numbers and domain positions are shown in Table 1. Because of the size difference of amino acid sequences in length among species, we have extracted commonly conserved sequence of 100 amino acids (designated 126-125) from each for reasonable comparison of RMSDs (Root Mean Square deviation). Multiple sequence alignment was performed using the ClustalW 1.83 program (Thompson et al., 1997) with default parameters

Table 1. Species name, PDB ID, and domain position of prion proteins.

\begin{tabular}{lcc}
\hline \multicolumn{1}{c}{ Species name } & PDB ID & Domain position \\
\hline Turtle & 1U5L & $121-226$ \\
Mouse & 1AG2 & $121-231$ \\
Bovine & 1DWY & $121-230$ \\
Human (no substitution) & 1HJM & $125-228$ \\
Human (E200K) & 1FKC & $90-231$ \\
\hline
\end{tabular}

A Multiple sequence alignment

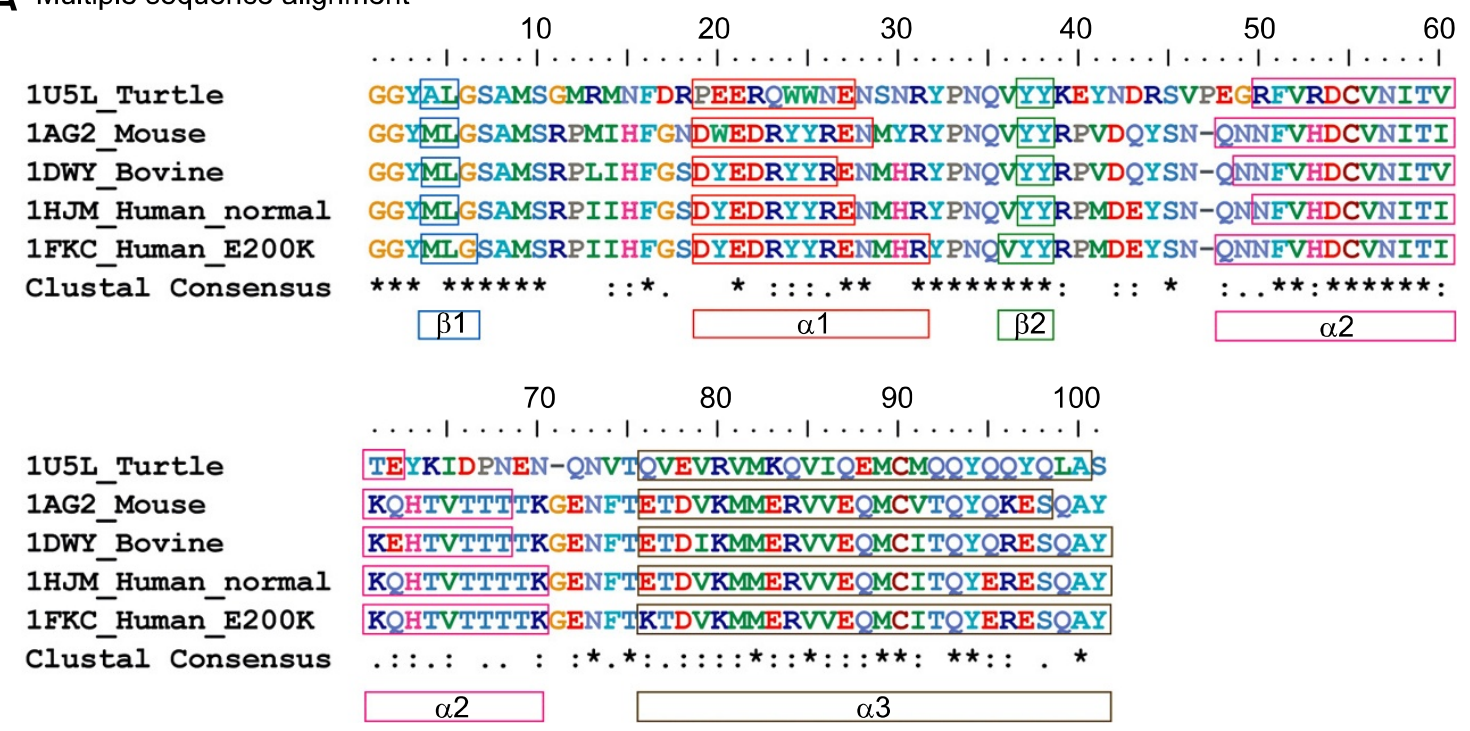

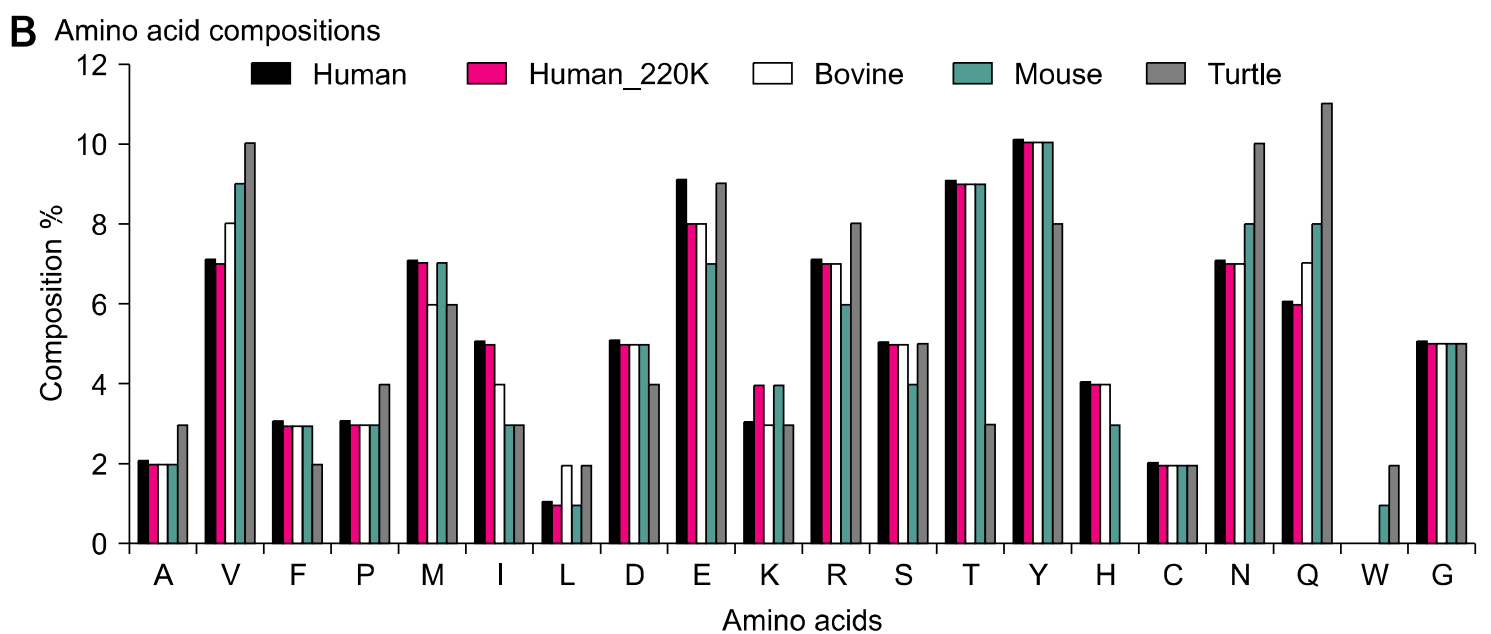

Figure 2. (A) Multiple sequence alignment, and (B) amino acid compositions of turtle, mouse, bovine, and human (normal and E200K) PrP amino acid sequences. The conserved regions of the secondary structural elements in the globular domain of the five proteins are represented as colored boxes (blue $=\beta 1$, red $=\alpha 1$, green $=\beta 2$, pink $=\alpha 2$, brown $=\alpha 3$ ). At bottom, asterisks indicate amino acids that are identical for the PrP sequences. Structural information for the secondary structure elements was collected from the PDB database. 
(gap-opening and -extension penalties of 10.0 and 0.20 , respectively). The Gonnet series matrix was used as the protein weight matrix. In addition, we also calculated amino acid compositions among PrPs from five species and the results are shown in Figure 2B.

\section{Molecular dynamics simulations}

Molecular dynamics (MD) simulations were performed using the program package AMBER 7 (Case et al., 2005) with the Amber 99 force field on an IBM p690 supercomputing system in Supercomputing Center, KISTI (Korea Institute of Science and Technology Information). To reduce computing time, we used the "Generalized Born" (GB) model, which efficiently describes the electrostatics of molecules in aqueous environments. It represents the solvent implicitly as a continuum with the dielectric properties of water and includes the charge-screening effects of salts. This approach generally hastens the simulation, an advantage that becomes increasingly important as the number of water molecules required in the explicit solvent simulation is increased. The basic algorithm of the GB model is:

$$
W_{\text {in }}=\frac{1}{2} \sum_{i \neq j} \frac{q_{i} q_{j}}{r_{i j}}-\frac{1}{2}\left(1-\frac{1}{\varepsilon_{\omega}}\right) \sum_{i, j} \frac{q_{i} q_{j}}{f^{g b}\left(r_{i j}\right)}
$$

where the dielectric constant of the solvent $\left(\varepsilon_{\omega}\right)$, and a certain smooth function $\left(f_{i j}^{a b}\right)$ is assumed to be dependent upon atomic radii $\left(\rho_{i}\right)$ and interatomic distances $\left(r_{i j}\right)$ (Bashford and Case, 2000). The first and second terms represent the energy in a vacuum and the solvation energy, respectively. In the second term, $f^{g b}\left(r_{i j}\right)$ is a function that interpolates between the effective Born radius $\alpha_{i}$ (when the distance $r_{i j}$ between atoms is short) and $r_{i j}$ itself (when the distance is large) (Still et al., 1990):

$$
f^{g b}\left(r_{i j}\right)=\left[r_{i j}^{2}+\alpha_{i} \alpha_{j} \exp \left(-r_{i j}^{2} / 4 \alpha_{i} \alpha_{j}\right)\right]^{1 / 2}
$$

The effective Born radius $\alpha_{i}$ represents the depth by which a charge is buried in a low-dielectric medium, such as a protein or nucleic acid (Srinivasan et al., 1999; Tsui and Case, 2001).

In the first step, AMBER topology and coordinate files were created by leap module using ff99 AMBER force field. Then, all of the structural abnormalities of the PrPs (the "hot spots" in the NMR structures), were eliminated by an energy-minimization process using the sander module of AMBER. Then, the system temperature was increased from $0 \mathrm{~K}$ to $300 \mathrm{~K}$ using the heating and equilibration processes with SHAKE algorithm. Finally, the MD simulation was conducted for $1,000,000$ cycles using a non-bonded cutoff value of $12 \AA$.

\section{RMSD and potential energy calculation}

The tertiary PrP structures resulting from each molecular simulation step were compared on the basis of RMS deviations calculated as:

$$
\text { R.M.S.D }=\sqrt{\frac{\sum_{i=1}^{N \text {.otatoms }}\left(r_{i}\left(t_{1}\right)-r_{i}\left(t_{2}\right)\right)^{2}}{N . \text { ofatoms }}}
$$

where $r_{i}(t)$ means the position of ith atom at time $t$. The program VMD (Visual Molecular Dynamics) v. 1.8.4 (Humphrey et al., 1996) was used to calculate RMS deviations (Figure 4). Potential energy of each PrPs along with time series was extracted from MD output files using perl script (Figure 3).

\section{Results}

\section{Phylogenetic relationships}

To investigate the phylogenetic relationships among rodent (group 1), reptile (group 2), ruminant (group

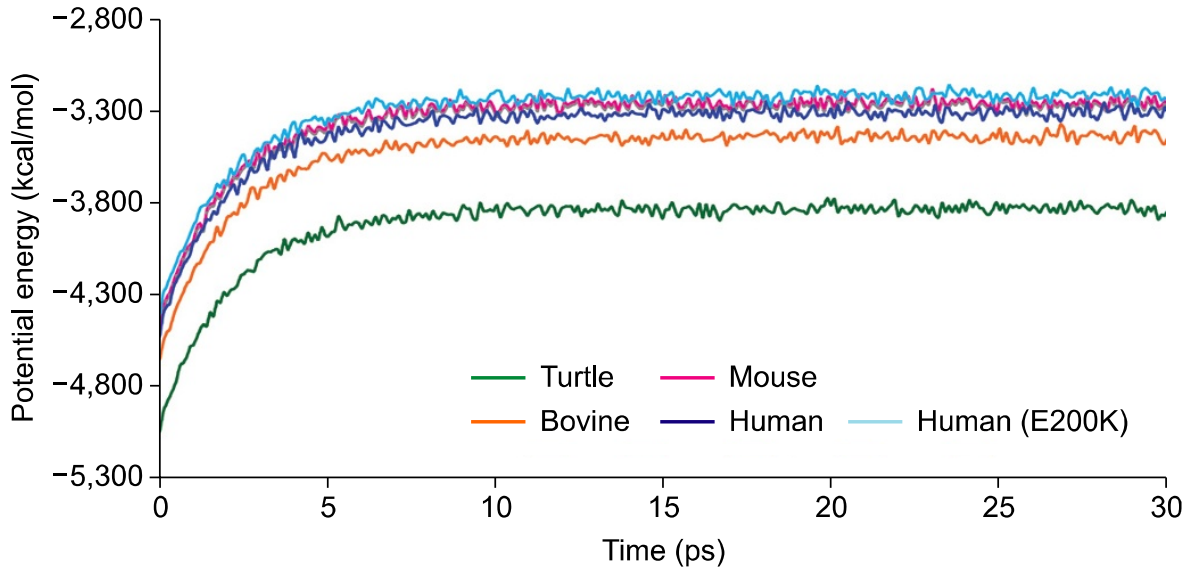

Figure 3. Change of potential energy values over simulation time during heating and equilibration stage. 


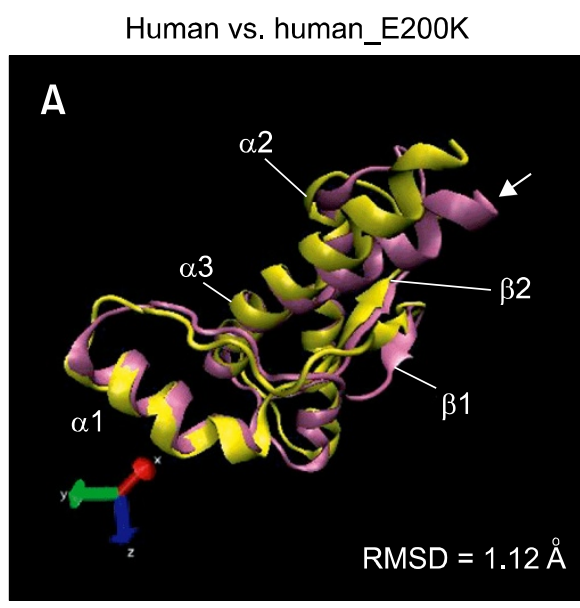

Human vs. mouse

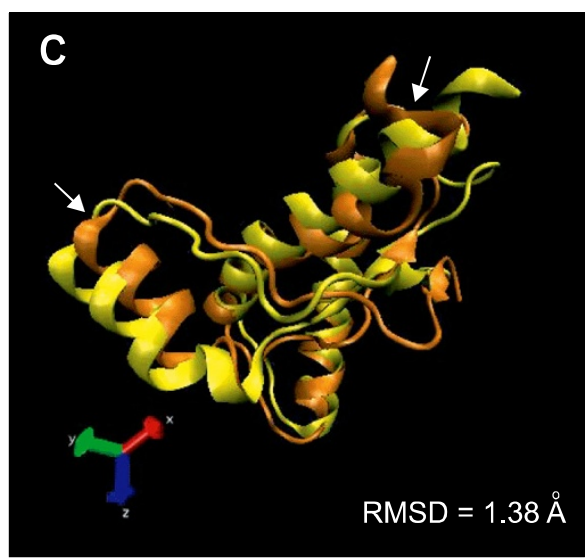

Human vs. bovine

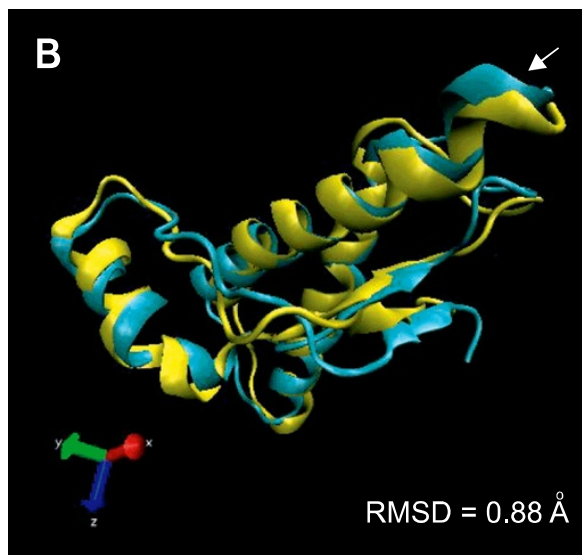

Human vs. turtle

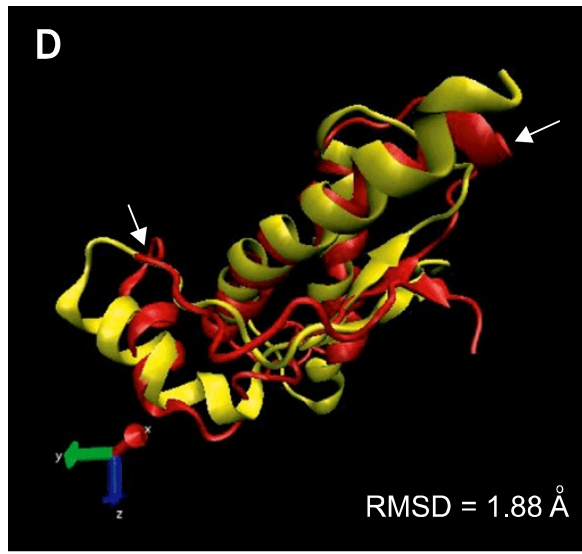

Figure 4. (A) Superimposed tertiary structures of the 126-225 polypeptide segments of normal human PrP (amino acids 125-228; yellow) and E200K human PrP (amino acids 90-231; mauve). A spline function was drawn through the $\mathrm{C}^{\alpha}$ positions. The radius of the cylindrical rods varies in proportion to the mean global backbone displacement per residue, as evaluated after superimposition for best fit of the atoms $\mathrm{N}, \mathrm{C}^{\alpha}$, and $\mathrm{C}^{\prime}$ in the two bundles of energy-minimized conformers used to represent the solution structure. (B-D) Tertiary structure of the 126-225 polypeptide segment of normal human PrP (amino acids 125-228) superimposed with those of the 126-225 polypeptide segments of (B) bovine (121-230; cyan), (C) mouse (121-231; orange), and (D) turtle (121-226; red) PrP. RMS deviations were calculated using VMD 1.8.4.
3) and human species (group 4), we created a phylogenetic tree using the Neighbor-Joining method (Figure 1). PrP sequences were clustered into four groups on the basis of their biological classes. Of these sequences, the one with the largest number of amino acid differences from the human (normal) PrP sequence was the turtle PrP sequence (branch length $=0.5751$ ). Among the 8 sequences, we chose 1 sequence from each class except for human cases, and then, used for the further analysis. The human E200K, bovine and mouse PrP sequences differed from that of normal human $\operatorname{PrP}$ with branch lengths of $0.0065,0.0826$ and 0.1032 , respectively. Thus, the distance between the normal human and turtle PrPs was about six times greater than that between the normal human and mouse PrPs.

\section{Secondary structure comparisons using multiple sequence alignment}

The major secondary structures (two $\beta$-sheets and three $\alpha$-helices), and the amino acid compositions of the PrP sequences are shown in Figure 2. Although the phylogenetic analysis described above showed that the turtle PrP amino acid sequence is the most distinct from other PrPs (Figure $1)$, it retained the major conserved regions of secondary structure found in the other species. The $\alpha 1$ - and $\alpha 2$ - helices of turtle PrP, however, were somewhat shorter than the corresponding elements of the other PrPs. The $\alpha 1$-helix and $\beta 1$ and $\beta 2$-strands of human E200K PrP were slightly longer than those of normal human PrP, based on the structural information found in the PDB database (Figure 2A). In the graph of amino acid compositions in Figure 2B, Y-axis means the amino acid composition (\%) in each PrP, and $\mathrm{X}$-axis represents each amino acid. Amino acids which have hydrophobic properties were showed first, from Alanine $(A)$ to Leucine $(L)$, and then, the charged amino acids were followed from aspartic acid (D) to arginine (R) on the X-axis. The polar amino acids were located from serine (S) to tryptophan (W), and glycine (G) was followed. Among hydrophobic amino acids, bovine, mouse and turtle 
PrPs showed higher composition in valine (V), whereas they were showed reverse composition in isoleucine (I). Turtle $\mathrm{PrP}$ also revealed distinct amino acid compositions in the polar amino acids such as threonine $(\mathrm{T})$, tyrosine $(\mathrm{Y})$, asparagine $(\mathrm{N})$ and glutamine $(\mathrm{Q})$. Moreover, turtle $\mathrm{PrP}$ revealed no histidine $(H)$, whereas it showed tryptophan (W) in its secondary structure. There was no tryptophan in human, human_200K and bovine PrPs.

\section{Comparison of potential energies}

Potential energies ( $\mathrm{kcal} / \mathrm{mol})$ for PrPs isolated from each species were analyzed by extracting the energy information from MD result files in heating (from $0 \mathrm{~K}$ to $300 \mathrm{~K}$ ) and equilibration $(300 \mathrm{~K}$ ) steps. All the changes of potential energy stage along with simulation time were presented by line graph in Figure 3. Pattern of energy increment of PrPs from 5 species revealed very similar and each PrP molecule showed fully equilibrated energy states. Their final energy states, however, were differed from each other. Firstly, turtle PrP showed the lowest potential energy state than other species, resulting near $-3,800 \mathrm{kcal} / \mathrm{mol}$, whereas other species showed somewhat upper levels between $-3,300$ and $-3,500 \mathrm{kcal} / \mathrm{mol}$. Among the rest of them, PrP from mouse species revealed more similar patterns with normal and E200K human PrPs than bovine PrP.

\section{Comparisons of RMS deviations}

RMS deviations between the structure of normal human PrP and the structures of the E200K human, bovine, mouse, and turtle PrPs were also calculated using the VMD program with energyminimized conformers representing the solution structure (Figure 4). The position of each secondary structure element (three $\alpha$-helices and two $\beta$-strands) is shown in Figure 4A, and the calculated RMS deviations are also shown in Figure 4. The largest RMS deviation from the human PrP structure was that of the turtle PrP structure (RMS $=1.88 \AA$ ), and the second largest was that of the mouse PrP structure (RMS $=1.38 \AA$ ) (Figure $4 \mathrm{C}$
Human (E200K)_initial

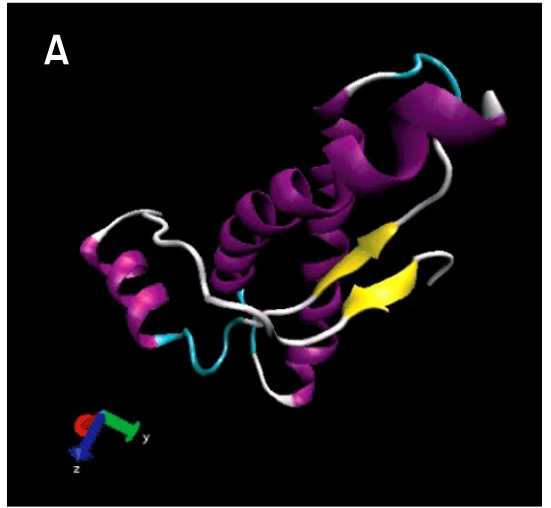

Human (E200K)_final

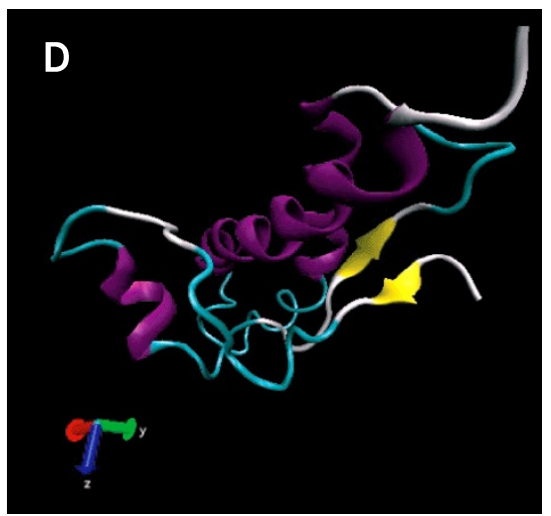

Bovine initial

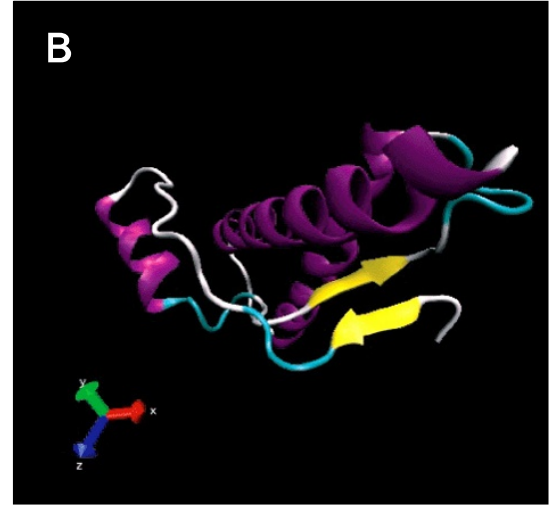

Bovine_final

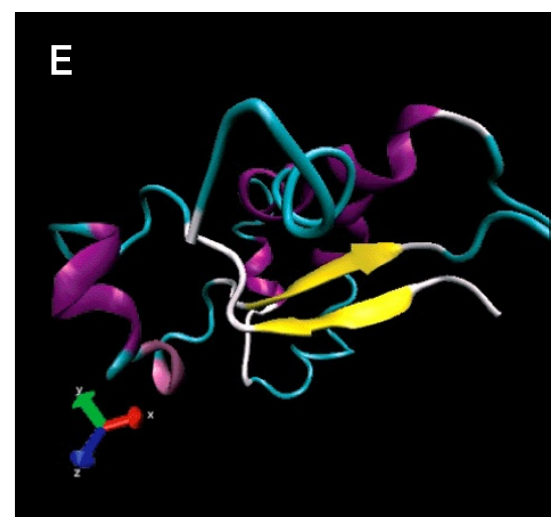

Turtle_initial

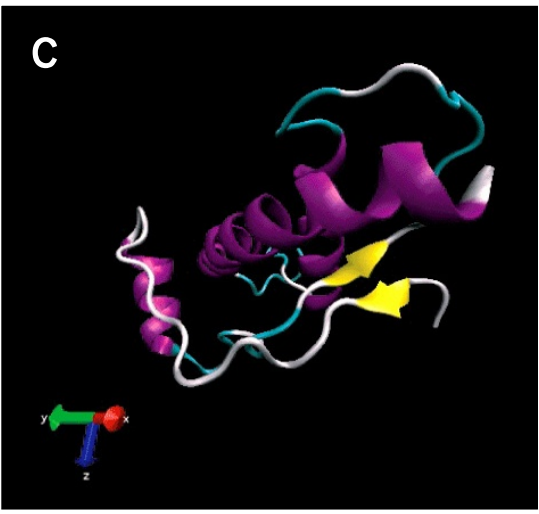

Turtle_final

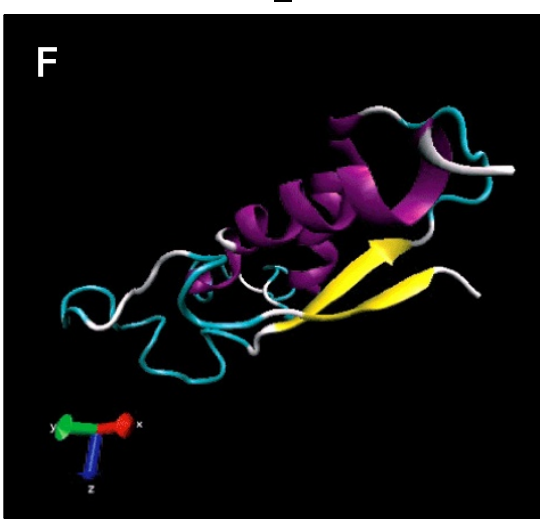

Figure 5. Predicted tertiary structures of human E200K, bovine, and turtle PrP. The initial (A-C) and final (D-F) structures resulted from MD simulations at $300 \mathrm{~K}$ are shown. 
and D).

Although the phylogenetic branch length between the normal and E200K human PrP sequences $(0.0065)$ was about one-thirteenth of that between the normal human and bovine PrPs $(0.0826)$ and potential energy state was more similar between the normal and E200K human PrPs than that of bovine PrP, the results of our structural analysis indicated a contrary result; compared with normal human PrP, the E200K human and bovine PrP RMS deviations were 1.12 and $0.88 \AA$, respectively (Figure $4 A$ and $B$ ). In the divergent $\alpha 1$-helix region, the structural difference between the E200K human and bovine PrPs was small (Figure 4A and B), whereas the structural difference between the mouse and turtle PrPs was relatively large (Figure $4 C$ and $D$ ). These regions are represented by short arrows in Figure 4. For the $\alpha 3$-helix of human E200K and bovine PrPs, the former was more divergent than the latter.

\section{Comparisons of molecular dynamics (MD) simulation results}

To investigate the structural differences among the MD simulation results for human E200K, bovine, and turtle PrPs, we compared the initial and final structures (Figure 5). The structures of the $\beta 1$ - and $\beta 2$-strands in the initial and final MD structures of each PrP were relatively conserved. The $\beta$-strands in the final bovine and turtle PrP structures were slightly longer than those of the corresponding initial structures, but those in the initial and final human E200K structures were of the same length (Figure 5D and E). The turtle $\operatorname{PrP} \alpha 1$-helix exhibited considerable deterioration in the final MD structure, whereas the $\alpha 1$-helices of the other PrPs were partially conserved in the final MD structure (Figure 5F). The $\alpha 3$-helix of bovine PrP near the C-terminus exhibited much larger changes than those of other PrPs (Figure 5E). The RMS deviations between the initial and final MD steps in the human E200K, bovine, and turtle PrP were $1.93,3.29$, and $1.96 \AA$, respectively.

\section{Discussion}

Since the pathogenic and normal cellular PrP molecules are alternative structural isoforms of the same peptide, without genetic alteration, molecular dynamics (MD) simulation offers an important tool in prion studies. Carrying out MD simulations in explicit solvent usually requires considerable computing time, even in a cluster or supercomputing system. In the present study, we determined the differences of the primary PrP sequences from 8 different species prior to the MD simulation first, using multiple sequence alignment and phylogenetic analysis techniques. And then, MD simulations using the Generalized Born model, which conducts the MD process in an implicit environment, were performed to identify the structural differences among PrPs. This combinational approach using different kinds of bioinformatics techniques is expected to give clearer information than that from single method.

As mentioned above, we conducted a phylogenetic analysis using 8 sequences such as turtle, mouse, hamster, bovine, sheep, elk and normal and E200K human PrPs to investigate the primary sequences of PrPs more intensively (Figure 1). All the PrPs were divided into four groups according to their biological classes, and turtle PrP was found to have the most distinct amino acid pattern among the PrPs analyzed. This result was also confirmed in the pattern of potential energy increment with showing the most distinct potential energy state of turtle PrP than any other sequences (Figure 3 ). In the analysis of the multiple sequence alignmennt, however, turtle PrPs showed five conserved secondary structural elements that are commonly found in other PrPs, suggesting that these regions are functionally important in vivo (Figure $2 \mathrm{~A}$ ). $\mathrm{PrP}^{\mathrm{C}}$ is usually expressed throughout the brain, particularly in neurons (Moser et al., 1995), and, to a lesser extent, in extraneural tissues (Ford et al., 2002). As for the amino acid properties, turtle PrP showed most distinct characteristics in the polar amino acid groups, especially in threonine $(T)$, tyrosine $(Y)$, asparagine $(\mathrm{N})$ and glutamine $(\mathrm{Q})$, and those differences might play an important role in secondary structure formation.

A comparison of the RMS deviation between the MD structures of normal and E200K human PrP with that between the structures of normal human and bovine PrPs yields an interesting result (Figure 4). Although the primary sequences of human and E200K PrP differ only in the $\mathrm{E} \rightarrow \mathrm{K}$ substitution at position 200 (Figure 2), our MD simulations yielded an RMS deviation for these two human PrPs that was slightly larger than that between normal human and bovine PrP. According to Zhang et al. (2000), the $E \rightarrow K$ substitution redistributes surface charges, resulting in a dramatically altered electrostatic potential in the mutant protein; this electrostatic change may have affected the PrP molecule during the energy-minimization process. In the MD simulations, the turtle PrP exhibited a distinct pattern of structural deterioration, as compared to the mammalian PrPs, with large structural changes in the $\alpha 1$-helix. Both $\beta$-strand structures were 
more stable than $\alpha$-helices 2 and 3 and exhibited little deterioration during the MD process. The pathogenic $\mathrm{PrP}^{\mathrm{Sc}}$, which is comparatively rich in $\beta$-sheet structure, is much more stable than $\operatorname{PrP}^{\mathrm{C}}$, exhibiting far less deterioration in the presence of UV rays, ionizing radiation, or high temperature.

In this study, we analyzed the primary and tertiary structural characteristics of various PrPs using bioinformatics techniques, including phylogenetic analysis and MD simulations. Phylogenetic analysis alone cannot always predict structural or functional differences between proteins correctly, as shown in the comparison between E200K human and bovine PrPs. The careful use of two or more bioinformatics tools in combination can produce better results than use of a single method alone; this combinatorial approach might be very helpful for thorough analysis of complex pathogens like PrP.

\section{Ackowledgments}

This work was supported by the Brain Korea 21 project in 2007.

\section{References}

Alpers MP. Epidemiology and ecology of kuru. In slow transmissible diseases of the nervous system, vol. 1, 1979, New York: Academic Press, NY, USA

Bashford D, Case DA. Generalized born models of macromolecular salvation effects. Annu Rev Phys Chem 2000;51: 129-52

Brown P, Goldfarb LG, Gibbs CJ Jr, Gajdusek DC. The phenotypic expression of different mutations in transmissible familial Creutzfeldt-Jakob disease. Eur J Epidemiol 1991;7: 469-76

Burns CS, Aronoff-Spencer E, Dunham CM, Lario P, Avdievich NI, Antholine WE, Olmstead MM, Vrielink A, Gerfen GJ, Peisach J, Scott WG, Millhauser GL. Molecular features of the copper binding sites in the octarepeat domain of the prion protein. Biochemistry 2002;41:3991-4001

Burns CS, Aronoff-Spencer E, Legname G, Prusiner SB, Antholine WE, Gerfen GJ, Peisach J, Millhauser GL. Copper coordination in the full-length, recombinant prion protein. Biochemistry 2003;42:6794-803

Case DA, Cheatham TE III, Darden T, Gohlke H, Luo R, Merz KM Jr, Onufriev A, Simmerling C, Wang B, Woods R. The Amber biomolecular simulation programs. J Comp Chem 2005;26:1668-88

Cathala F, Brown P, LeCanuet P, Gajdusek DC. High incidence of Creutzfeldt-Jakob disease in North African immigrants to France. Neurology 1985;35:894-5

Dawson M, Wells GAH, Parker BNJ. Preliminary evidence of the experimental transmissibility of bovine spongiform encephalopathy to cattle. Vet Rec 1990;126:112-3

Fraser H, Bruce ME, Chree A, McConnel I, Wells GA. Transmission of bovine spongiform encephalopathy and scrapie to mice. J Gen Virol 1992;73:1891-7

Ford MJ, Burton LJ, Morris RJ, Hall SM. Selective expression of prion protein in peripheral tissues of the adult mouse. Neuroscience 2002;113:177-92

Gajdusek DC. Unconventional viruses and the origin and disappearance of kuru. Science 1977;197:943-60

Hadlow WJ, Kennedy RC, Race RE. Natural infection of Suffolk sheep with scrapie virus. J Infect Dis 1982;146: 657-64

Hill AF, Desbrusliers M, Joiner S, Sidle KCl, Gowland I, Collinge J, Doey LJ, Lantos PS. The same prion strain cuases CJD and BSE. Nature 1997;389:448-51

Humphrey W, Dalke A, Schulten K. VMD_Visual Molecular Dynamics. J Molec Graphics 1996;14:33-8

Jackson GS, Murray I, Hosszu LL, Gibs N, Waltho JP, Clarke AR, Collinge J. Location and properties of metal-binding site on the human prion protein. Proc Natl Acad Sci 2001;98: 8531-5

Kahana E, Milton A, Braham J, Sofer D. Creutzfeldt-Jakob disease: Focus among Libyan Jews in Israel. Science 1974; 183:90-1

Kirkwood JK, Cunningham AA. Epidemiological observations on spongiform encephalopathies in captive wild animals in the British Isles. Vet Rec 1994;135:296-303

Klazo I, Gajdusek DC, Zigas V. Pathology of krur. Lab Invest 1959;8:799-847

Kramer ML, Kratzin HD, Schmidt B, Romer A, Windl O, Liemann S, Hornemann S, Kretzschmar H. Prion protein binds copper within the physiological concentration range. J Biol Chem 2001;276:16711-9

Lasmézas Cl, Deslys J-P, Demalmay R, Adjou KT, Lamoury F, Dormont D, Robain O, Ironside JW, Hauw JJ. BSE transmission to macaques. Nature 1996;381:743-4

Moser M, Colello RJ, Pott U, Oesch B. Developmental expression of the prion protein gene in glial cells. Neuron 1995;14:509-17

Nisipeanu P, El AdB, Korczyn AD. Spongiform encephalopathy in an Israeli born to immigrants from Libya (letter). Lancet 1990;336:686

Page RDM. TREE VIEW: An application to display phylogenetic trees on personal computers. Comp Applic Biosci 1996;12:357-8

Parchi P, Giese A, Capellari S, Brown P, Schulz-Schaeffer W, Windl O, Zerr I, Budka, H, Kopp N, Piccardo P, Poster S, Rojiani A, Streichemberger N, Julien J, Vital C, Ghetti B, Gambetti P, Kretzschmar H. Classification of sporadic Creutzfeldt-Jakob disease based on molecular and phenotypic analysis of 300 subjects. Ann Neurol 1999;46: 224-33

Prusiner SB. Prion biology and diseases: second edition, 2004, Cold Spring Harbor Laboratory Press, NY, USA 
Qin K, Yang Y, Mastrangelo P, Westaway D. Mapping Cu(II) binding sites in prion proteins by diethyl pyrocarbonate modification and matrix-assisted laser desorption ionization-time of flight (MALDI-TOF) mass spectrometric footprinting. J Biol Chem 2002;277:1981-90

Simon ES, Kahana E, Chapman J, Treves TA, Gabizon R, Rosenmann H, Zilber N, Korczyn AD. Creutzfeldt-Jakob disease profile in patients homozygous for the PRNP E200K mutation. Ann Neurol 2000;47:257-60

Srinivasan J, Trevathan MW, Beroza P, Case DA. Application of a pairwise Generalized Born model to proteins and nucleic acids: inclusion of salt effects. Theor Chem Acc 1999;101: 426-34

Still WC, Tempczyk A, Hawley RC, Hendrickson T. Semianalytical treatment of salvation for molecular mechanics and dynamics. J Am Chem Soc 1990;112:6127-9

Swofford DL. PAUP*. Phylogenetic Analysis Using Parsimony ( ${ }^{*}$ and Other Methods). Version4. 1999, Sinauer, Sunderland, MA

Thompson JD, Gibson TJ, Plewniak F, Jeanmougin F,
Higgins DG. CLUSTALX windows interface: flexible strategies for multiple sequence alignment aided by quality analysis tools. Nucleic Acids Res 1997;25:4876-82

Tsui V, Case DA. Theory and applications of the Generalized Born salvation model in macromolecular simulations. Biopolymers 2001;56:275-91

Wells GA, Scott AC, Johnson CT, Gunning RF, Hancock RD, Jeffrey $M$, Dawson $M$, Bradley R. A novel progressive spongiform encephalopathy in cattle. Vet Rec 1987;121: 419-20

Wells GAH, Hawkins SAC, Austin AR, Ryder SJ, Done SH, Green RB, Dexter I, Dawson M, Kimberlin RH. Studies of the transmissibility of the agent of bovine spongiform encephalopathy to pigs. J Gen Virol 2003;84:1021-31

Zhang Y, Swietnicki W, Zagorski MG, Surewicz WK, Sonnichsen FD. Solution structure of the E200K variant of human prion protein. J Biol Chem 2000;275:33650-4

Zilber N, Kahana E, Abraham MPH. The Libyan CreutzfeldtJakob disease focus in Israel: An epidemiologic evaluation. Neurology 1991;41:1385-9 